ARTICLE

\title{
Directed manipulation of membrane proteins by fluorescent magnetic nanoparticles
}

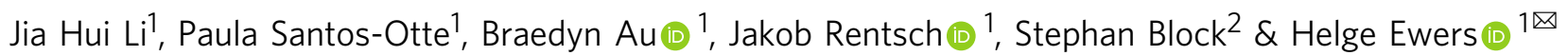

The plasma membrane is the interface through which cells interact with their environment. Membrane proteins are embedded in the lipid bilayer of the plasma membrane and their function in this context is often linked to their specific location and dynamics within the membrane. However, few methods are available to manipulate membrane protein location at the single-molecule level. Here, we use fluorescent magnetic nanoparticles (FMNPs) to track membrane molecules and to control their movement. FMNPs allow single-particle tracking (SPT) at $10 \mathrm{~nm}$ and $5 \mathrm{~ms}$ spatiotemporal resolution, and using a magnetic needle, we pull membrane components laterally with femtonewton-range forces. In this way, we drag membrane proteins over the surface of living cells. Doing so, we detect barriers which we could localize to the submembrane actin cytoskeleton by super-resolution microscopy. We present here a versatile approach to probe membrane processes in live cells via the magnetic control of membrane protein motion.

\footnotetext{
${ }^{1}$ Institut für Biochemie, Freie Universität Berlin, Thielallee 63, 14195 Berlin, Germany. ${ }^{2}$ Institut für Chemie, Freie Universität Berlin, Takustr. 3,14195 Berlin, Germany. ${ }^{凶}$ email: helge.ewers@fu-berlin.de
} 
$\mathrm{M}$ embrane proteins are heterogeneously distributed in the plasma membrane of cells despite its fluid and continuous nature ${ }^{1,2}$. Local accumulation or separation of specific membrane molecules is crucial for efficient execution and regulation of fundamental biological processes, such as (synaptic) signaling $^{3}$, cellular secretion ${ }^{4}$ and internalization of nutrients $s^{5}$, cellular movement ${ }^{6}$, and tissue formation ${ }^{7}$. Imaging techniques such as super-resolution imaging, SPT, and fluorescence correlation spectroscopy have been instrumental to describe the local membrane composition and dynamics ${ }^{8}$. To perturb the functional localization and dynamics of a given membrane molecule, genetic and pharmacological means are usually chosen. However, these approaches lead to global effects and are very limited in temporal resolution. Optogenetics and other photoactivation methods enable better temporal control but nevertheless lack spatial information at the single-molecule level. To directly move membrane molecules, optical tweezers ${ }^{9,10}$ have been used, but this method requires sophisticated equipment and mostly allows the control of only one particle at a time.

Here, we sought to use magnetic nanoparticles as a straightforward alternative. Magnetic nanoparticles are widely used in biomedicine for imaging and therapy ${ }^{11,12}$. In biophysics, magnetic tweezers with micrometer-sized magnetic beads are established tools for force spectroscopy and microrheology ${ }^{13-16}$. In the last decade, advances in the use of biofunctionalized magnetic particles to remotely control cellular processes have opened a new field termed "magnetogenetics"17. Micro- to nanometer-sized magnetic particles coated with active biomolecules are introduced to the specimen to actuate mechanical, thermal, or biochemical signals. Functionalized magnetic beads could be moved and tracked in the cytosol ${ }^{18-20}$. At the plasma membrane, mechano-, heat-, or clustering-sensitive receptors and ion channels could be activated using magnetic particles ${ }^{21-23}$. Usually, either large clusters or micrometer-sized beads or a high concentration of particles are used for ensemble measurements. In contrast, we here use particles sized below the diffraction limit of light in a low-density regime to retrieve information on single molecules with high spatiotemporal resolution. The magnetic component of the particles then allows concomitant manipulation by applying an external magnetic field. Our method provides spatial control over membrane protein motion while determining its localization with nanometer precision. This allows for the exact correlation of membrane protein location with cellular events and structures.

\section{Results}

Magnetic control over membrane lipid diffusion. First, we aimed to determine whether FMNPs were compatible with SPT and magnetic manipulation in the focal plane of a fluorescence microscope in aqueous conditions. To do so, we made use of particles consisting of a $100 \mathrm{~nm}$ diameter ferromagnetic core and a polymer shell conjugated with a fluorescent dye and streptavidin. We found these particles to be monodisperse in aqueous buffer and brightly fluorescent. The FMNPs bound readily to supported lipid bilayers (SLB) containing $0.001 \mathrm{~mol} \%$ biotinylated lipids and were detectable as single, diffraction-limited spots (Fig. 1a). When we tracked the particles over time at a frame rate of $50 \mathrm{~Hz}$ in TIRF illumination, we found that they exhibited random motion in the plane of the membrane with a mean/ median diffusion coefficient $D$ of $0.13 / 0.15 \mu \mathrm{m}^{2} \mathrm{~s}^{-1}$ (931 tracks; Fig. 1b, left; Supplementary Movie 1). When we then approached a magnetized needle ${ }^{19}$ to the field of view using a micromanipulator, the particles started to move towards the tip of the needle (Fig. 1b, middle; Supplementary Movie 1). Upon removal of the needle, the particles returned to random motion (Fig. 1b, right; Supplementary Movie 1). We concluded that we could pull lipids in the plane of the membrane into the direction of the needle via FMNPs and that we could do so in a reversible and temporally controlled manner.

The particle movement under magnetic attraction results from the directed motion towards the magnetic needle and the inherent Brownian motion. To estimate the forces that are exerted on the FMNP-bound phospholipid by the magnetic needle, we separated the components of the trajectory indirection-of (directed component) and perpendicular-to (Brownian component) the needle tip (Fig. 1b). Using Eq. (1), we directly calculate the force from each trajectory of hundreds of particles bound to lipids in the SLB (Fig. 1c) ${ }^{24}$.

$$
F=k_{\mathrm{B}} T \frac{v_{x}}{D}
$$

The average velocity $v_{x}$ can be extracted from the linear movement in the direction of the magnetic field ( $x$ axis) and the average FMNP diffusion coefficient $D$ from the fluctuations in the direction perpendicular to the magnetic field ( $y$ axis). The calculated forces in $x$ direction are $13.4 \pm 12.9 \mathrm{fN}$ (mean \pm standard deviation, 711 tracks) when the magnetic field is applied and are undetectable without the magnet $(0.5 \pm 9.8 \mathrm{fN}, 178$ tracks; Fig. 1d, inset). The apparent force decreases with increasing distance from the tip, however above $100 \mu \mathrm{m}$, the distance dependency is obscured due to noise (Fig. 1d).

Notably, both the directed and random movement components are apparent in the trajectories (Fig. 1b), i.e., the displacement per frame due to the applied force is comparable to the one due to Brownian motion. The energy which is spent to drag the molecule through the lipid bilayer against friction is therefore on the order of the thermal energy $k_{\mathrm{B}} T$. This is desirable when applied to membrane proteins on living cells as forces in the femtonewton range will not abrogate most naturally occurring interactions of membrane proteins ${ }^{25}$. Instead, the magnet-attracted membrane protein can probe its path for functional interactions in the membrane environment. We concluded that FMNPs work as a straightforward probe to track membrane-bound molecules and to move them in a controlled, directed manner.

To estimate the localization precision we achieved in fluorescence microscopy, we imaged immobile FMNPs on the SLB and localized them over thousands of subsequent frames. Because of the high fluorescence brightness, the position center could be determined with high precision by sub-pixel localization. We found that FMNPs could be localized with $10 \mathrm{~nm}$ precision (Fig. 2a), and this was possible at frame rates down to $5 \mathrm{~ms}$ and up to 10,000 frames. To demonstrate this also for moving particles, we took advantage of structural inhomogeneities of the SLBs that were created by scratching the cover glass resulting in a gap in the continuous lipid bilayer. When we pulled FMNPs attached to biotinylated lipids in the SLB against such defects, we found that they appeared to slide along the defect boundaries (Fig. 2b). Since the FMNPs can be localized with nanometer localization precision, they map the boundaries of the SLB significantly more accurate than the diffraction-limited image of the SLB (image resolution $>200 \mathrm{~nm}$, Fig. 2c).

Magnetic control of membrane protein motion on living cells. We next aimed to test whether our system could be applied to living cells. To do so, we coupled FMNPs to commonly studied plasma membrane proteins transiently expressed in living mammalian cells. We expressed a probe for the outer leaflet of the plasma membrane, glycosylphosphatidylinositol-anchored green fluorescent protein (GPI-GFP), and two transmembrane probes, the yellow fluorescent protein-tagged single-spanning transmembrane domain of the low-density lipoprotein-receptor (LYFP-GT46) and the GFP-tagged full-length transferrin-receptor 
a
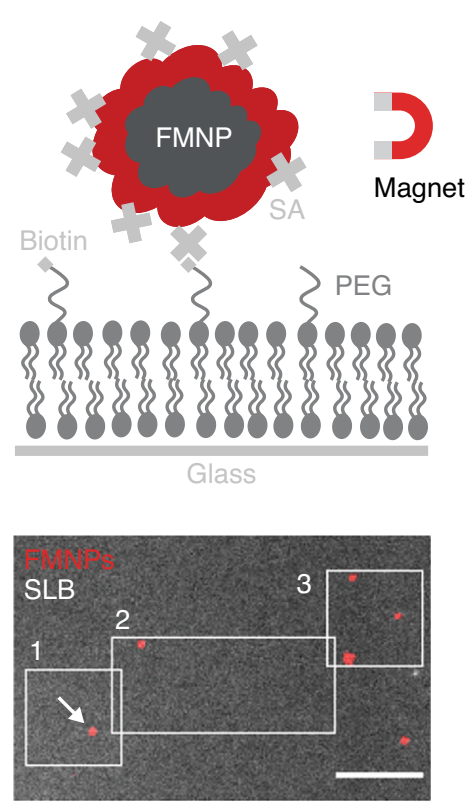

b

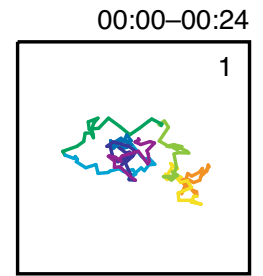

Before magnet

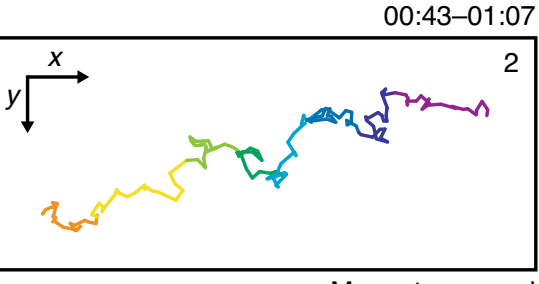

Magnet engaged

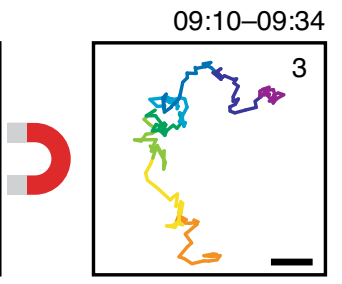

Magnet removed

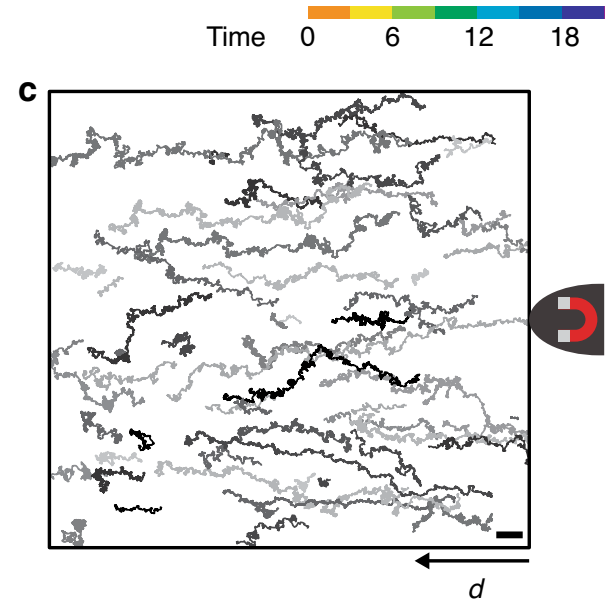

Fig. 1 Manipulation of lipids by FMNPs on an SLB and force calibration. a Experimental setup for the directed manipulation of a streptavidin (SA)functionalized FMNP bound to biotinylated lipid (DSPE-PEG(2k)-biotin) in a SLB. Graphic is not to scale. Below: Overlay of the first frame of a fluorescence time-lapse video of FMNPs (red) bound to a SLB doped with carboxyfluorescein-conjugated lipid (DOPE-CF, gray). Scale bar is $10 \mu \mathrm{m}$. $\mathbf{b}$ Trajectory of the FMNP marked with an arrow in a before (1), during magnetic manipulation (2) and afterwards (3) colored by time. Scale bar is $2 \mu \mathrm{m}$. c Trajectories of FMNPs tracked on a SLB with the very tip of the magnetic needle placed to the right. A subset of all tracks is shown in random gray levels for clarity. d Distance dependency of the apparent force. Forces were calculated for sliding windows of each trajectory in the data set in $\mathbf{c}$ and mapped to the average distance $d_{x}$ from the tip in direction of $x$. Inset: Histogram of apparent forces $F_{x}$ per trajectory exerted on FMNPs in presence (magenta, 711 tracks) and absence (blue, 178 tracks) of the magnetic needle. Source data are provided as a Source Data file.

(TfR-GFP). To deliver the FMNPs to the target probe, we used biotinylated anti-GFP nanobodies, which bind to GFP and derived variants such as YFP (Fig. 3a). The nanobody-conjugated FMNPs bound specifically on the dorsal membrane of transfected cells (Supplementary Fig. 1e) and single, mobile FMNPs could readily be tracked. When we approached the sample with the magnetic needle, the outer-leaflet probe as well as the transmembrane proteins could be moved over several micrometers in the plane of the plasma membrane towards the needle (Fig. 3b) with a velocity of $0.23 \pm 0.18 \mu \mathrm{m}^{2} \mathrm{~s}^{-1}(n=9)$. Freely diffusing particles exhibited no detectable change in diffusion coefficients when they resumed random motion after being dragged across the cell $\left(0.046 / 0.027 \mu \mathrm{m}^{2} \mathrm{~s}^{-1}\right.$ before, $0.040 / 0.027 \mu \mathrm{m}^{2} \mathrm{~s}^{-1}$ after magnetic dragging (mean/median; n.s., Student's $t$-test)). We concluded that we could track and manipulate proteins with different membrane anchoring moieties on living cells using FMNPs.

Interaction of membrane proteins with local structures. We then aimed to correlate the motion of membrane proteins with the location of cellular structures. In living cells, the cortical cytoskeleton affects the movement of membrane lipids and proteins ${ }^{26-32}$. Cytoskeletal actin-protein polymers form a dense meshwork underneath the plasma membrane ${ }^{33}$. To ask whether we could detect a physical barrier to membrane protein motion formed by actin filaments (F-actin), we pulled GPI-GFP molecules over the surface of CV-1 cells, fixed them and subsequently performed biplane 3D (d)STORM super-resolution microscopy of F-actin via phalloidin-AF647. This allowed us to specifically visualize cortical F-actin at the dorsal membrane (Supplementary Fig. 2). We then used fiducial markers present both in the SPT and (d)STORM experiments to overlay the SPT trajectory with the (d)STORM super-resolution image of the cortical F-actin. Doing so, we could detect that a GPI-GFP molecule, which was dragged for $>2 \mu \mathrm{m}$ across the cell membrane, slowed down and came to a halt (Fig. 4a; Supplementary Movie 2). The particle did not move directly towards the needle but seemed to avoid sites of dense F-actin (Fig. 4b). It then slowed down as it approached a thick, bundled actin filament and slid along this filament in direction of the needle. This movement pattern suggests that the GPI-GFP either experienced a physical barrier, directly imposed by the actin filament or indirectly by actin-associated transmembrane proteins or extracellular matrix, or a specific interaction with a cellular structure such as a clathrin-coated pit. On a broader scope, this experiment demonstrates that sites of interaction can be detected immediately and dynamically by introducing a directed movement component to the diffusional motion. Although classical SPT experiments also allow the exploration of the local membrane structure, due to the random nature of molecular diffusion, robust conclusions can only be drawn given high statistical sampling in time and space. In contrast, our approach makes it possible to directly probe the interaction of a membrane molecule with specific cellular structures and to obtain meaningful results for each individual particle.

\section{Discussion}

By using FMNPs, we have here combined the non-invasive perturbation capabilities of magnetic fields with high-precision SPT 
a

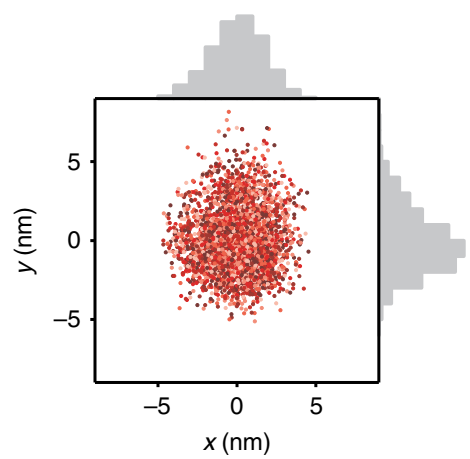

b

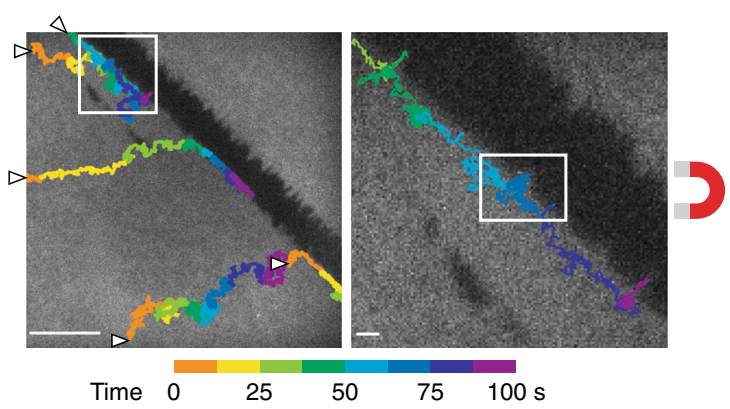

C
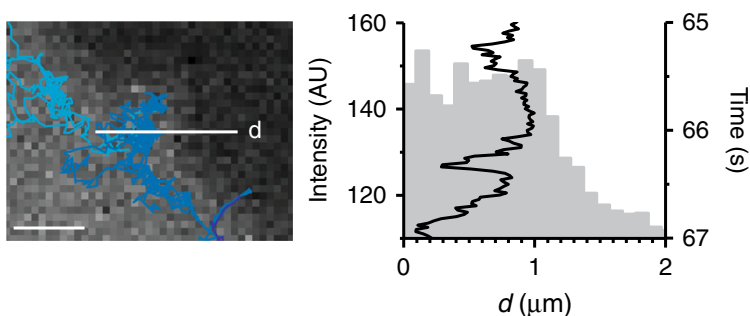

Fig. 2 Accuracy of FMNP localization and tracking. a Plot of subsequent 2D localizations of a single immobile FMNP on a SLB by sub-pixel fitting in each frame (total 3000 localizations, imaged at $50 \mathrm{~Hz}$ ), colored by time (light to dark red). $\mathbf{b}$ Trajectories of SLB-bound FMNPs under magnetic pull (beginning marked with arrowheads) overlaid onto the fluorescence image of the SLB (gray) with defects (black areas). Scale bars are $10 \mu \mathrm{m}$ and $1 \mu \mathrm{m}$. c Fluorescence micrograph of inset in $\mathbf{b}$ and plot of FMNP localization in direction of the white line (d) over time above plot of SLB fluorescence intensity along (d). Scale bar is $1 \mu \mathrm{m}$. Source data are provided as a Source Data file.

of a molecule of interest. Compared to optical tweezers, our approach is straightforward, low-cost, and allows to move several FMNPs at the same time. Previous ensemble applications of magnetic nanoparticles demonstrated the potential of local control over cellular processes ${ }^{19,21}$. We have occasionally observed that two particles aggregated under magnetization, therefore the particle density must be considered. In previous work, higher particle densities have indeed been employed to aggregate membrane proteins in a controlled manner, e.g., to elicit receptor signaling 21,34 . A further common issue of using nanoparticles for SPT is potential crosslinking of the targeted membrane molecules through multiple binding sites on the nanoparticle ${ }^{35,36}$, which could lead to unwanted effects depending on the target molecule. For the live-cell experiments, we addressed this by using a monovalent nanobody at a 1:1 stoichiometry per particle. We could not detect any effect on particle diffusion over time, however, we cannot exclude residual paucivalent binding. Due to our lateral magnetic tweezer setup, the pulling force can be applied in the focal plane of conventionally used inverted microscopes, rendering it compatible with many imaging approaches ${ }^{37}$. Our present work combines magnetic control of molecule movement with fluorescence-based SPT. This approach could be extended to other, non-fluorescent SPT techniques such as interferometric scattering microscopy (iSCAT) ${ }^{38}$ for even faster measurements. Furthermore, we demonstrate the compatibility with other imaging techniques by performing post-hoc super-resolution imaging of the cytoskeleton. In principle, the magnetic manipulation could be performed concomitantly with other live super-resolution imaging methods such as photoactivated localization (PALM) or live-cell stimulated emission depletion (STED) to capture the dynamic remodeling of the cellular environment. The combination of these techniques enables the interrogation of membrane components and their local environment at spatiotemporal scales that match their activity. In this context, exerting only small forces on the molecule of interest is crucial to maintain the potential for productive intermolecular interactions. In the future, this method could be applied to study receptor signaling at the plasma membrane. One could extract a specific component of a signaling hub and monitor the downstream effects in real time. Receptor dimerization for example is an important but hardly tractable problem in signaling. By pulling via an FMNP on one receptor subunit and simultaneously imaging the movement of the other subunit, quantitative data on the dimerized fraction of receptors in the membrane should be instantly accessible. Another field of application could be the investigation of polarized cells in which different membrane domains have a distinct functional organization and composition. A molecule of interest could be moved to an ectopic location on the membrane to study its context-dependent activity. Combining SPT and magnetic manipulation expands our current toolbox of methods to observe and to perturb the location of molecules in the plasma membrane. Our work thus opens the doors to easily accessible experiments aimed at understanding the interaction of membrane molecules with specific membrane-apposed cellular structures.

\section{Methods}

Nanoparticle characterization and magnetic tweezer setup. $100 \mathrm{~nm} \mathrm{BNF-}$ Starch-redF particles with streptavidin surface (Micromod) are composed of a magnetite core and a starch shell crosslinked with the red fluorescent dye Dy555 (Supplementary Fig. 1b). They are thermally blocked at room temperature. Dynamic light scattering (DLS) measurements with a $633 \mathrm{~nm}$ laser (Zetasizer Nano, Malvern) of the particles diluted 1:500 in phosphate buffered saline (PBS) confirmed that they are monodisperse with a polydispersity index of $0.11(<0.2$ is considered monodisperse) and a hydrodynamic radius of $159 \pm 2 \mathrm{~nm}$ ( $Z$-average size, Supplementary Fig. 1a). The magnetic needle $e^{19,20}$ is made of a spring steel wire of $0.1 \mathrm{~mm}$ diameter (Fohrmann-Werkzeuge), which was pulled in a Bunsen flame to create a tip (Supplementary Fig. 1d). The needle was then glued to a $6 \times 4$ $\times 2 \mathrm{~mm}$ Neodyn magnet (QM-06x04x02-N, Magnets4you) and attached to a motorized micromanipulator (PatchMan, Eppendorf). The magnetic needle was lowered until it touched the bottom of a dummy sample dish and raised again so that it was placed above the bottom of the sample with the very tip in focus around $100 \mu \mathrm{m}$ above the focus of the glass surface (Supplementary Fig. 1c).

SLB preparation and particle binding. Small unilamellar vesicles were prepared by sonication and used to form SLBs by the vesicle drop method. DOPC $(1,2-$ dioleoyl-sn-glycero-3-phosphocholine), DSPE-PEG(2k) (1,2-distearoyl-sn-glycero3-phosphoethanolamine- $N$-[carboxy(polyethylene glycol)-2000]), DSPE-PEG(2k) biotin (1,2-distearoyl-sn-glycero-3-phosphoethanolamine- $N$-[biotinyl(polyethylene glycol)-2000]) and DOPE-CF (1,2-dioleoyl-sn-glycero-3-phosphoethanolamine- $N$ carboxyfluorescein) were purchased from Avanti Polar Lipids. DOPC doped with $0.2-2 \mathrm{~mol} \%$ PE-CF and 1-2 mol\% of either DSPE-PEG(2k) or DSPE-PEG(2k)-

biotin were dissolved in 1:1 chloroform:methanol and dried under vacuum overnight. The lipids films were hydrated in SLB buffer $\left(2 \mathrm{mM} \mathrm{CaCl}_{2}, 200 \mathrm{mM} \mathrm{NaCl}\right.$, and $10 \mathrm{mM}$ HEPES, pH 6.8; all Sigma Aldrich), sonicated for $50 \mathrm{~s}$ and centrifuged for $30 \mathrm{~min}$ at top speed. The supernatants were stored at $4{ }^{\circ} \mathrm{C}$ for up to 3 weeks until use. Round $25 \mathrm{~mm} \# 1.5$ coverslips (VWR) or $35 \mathrm{~mm}$ diameter glass-bottom dishes (Zell-Kontakt) were plasma cleaned for $5 \mathrm{~min}$ prior to SLB formation. Solutions of DSPE-PEG(2k)- and DSPE-PEG(2k)-biotin-containing vesicles were mixed in appropriate concentration ratios to achieve a final concentration of 0.001-0.005 mol\% DSPE-PEG(2k)-biotin in the solution and dropped directly onto the glass or into a 4 -well silicon mold (Ibidi) on the glass. The bilayer was washed 
a

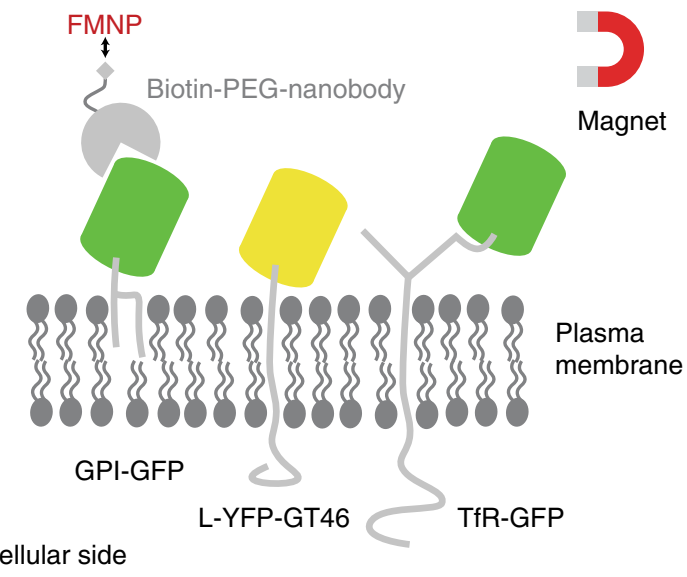

GPI-GFP

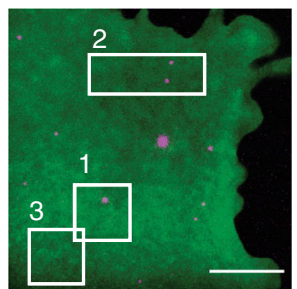

Intracellular side
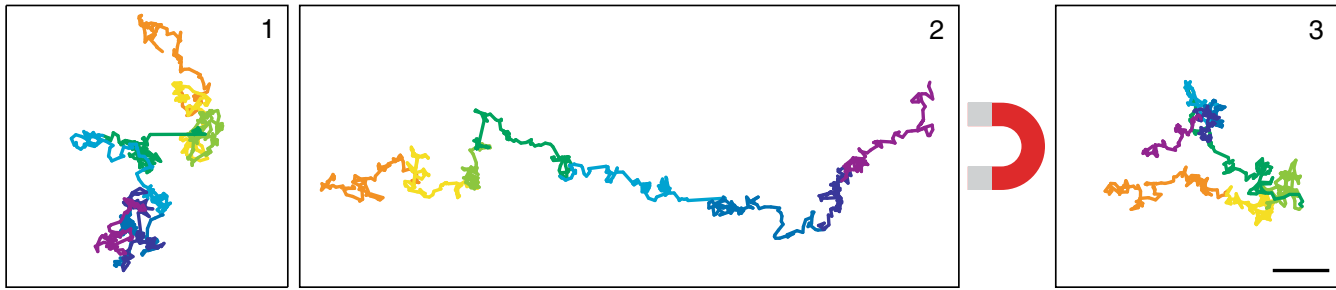

L-YFP-GT46
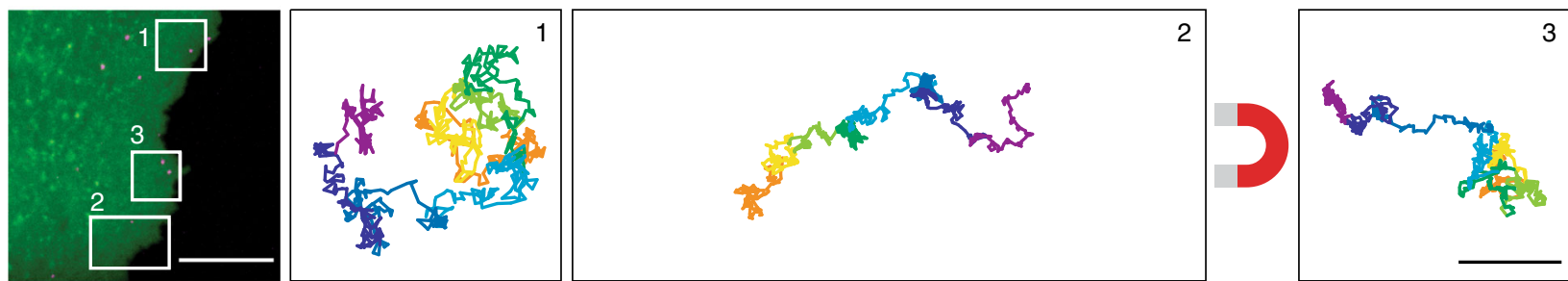

TfR-GFP
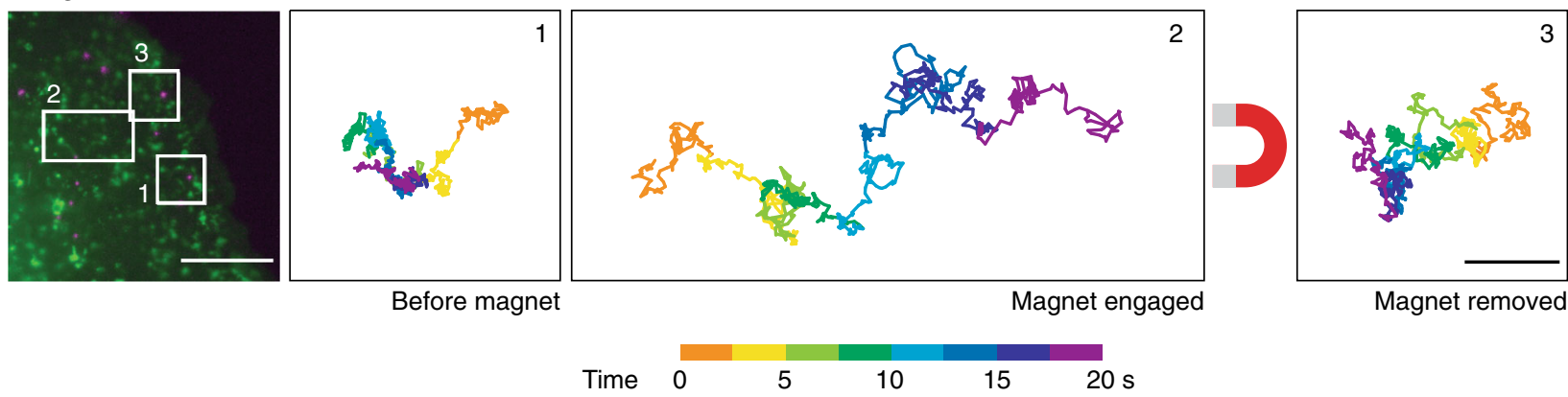

Fig. 3 Manipulation of membrane proteins by FMNPs on the surface of living cells. a Scheme of the manipulation of the outer leaflet lipid-anchored (GPIGFP) and transmembrane protein probes (L-YFP-GT46 and TfR-GFP) in living cells. The FMNPs are targeted to the fluorescent protein moiety of membrane protein probes via biotinylated anti-GFP nanobodies and then pulled across the plasma membrane by a magnetic needle. Graphic is not to scale. b From top to bottom: Experiments of membrane protein probes pulled across the plasma membrane of living CV-1 cells expressing GPI-GFP, L-YFP-GT46, or TfR-GFP. Left: Fluorescence micrographs of single cells expressing the respective constructs (green) with bound FMNPs (magenta). Scale bars are $10 \mu \mathrm{m}$. Right: Trajectories of exemplary FMNPs that moved in the white-framed areas on the cell before (1), during (2) and after (3) magnet engagement. Trajectories are color coded for time. Scale bars are $1 \mu \mathrm{m}$.

ten times with SLB buffer and evaluated on the spinning disc confocal microscope by fluorescence recovery after photobleaching. SLBs were incubated with $1 \times 10^{9}$ per ml FMNPs in SLB buffer with $1 \%$ BSA on the microscope until tens to hundreds of particles per field of view had bound to the SLB, and then washed 5-10 times with SLB buffer before imaging.

Cell culture. Wild-type Cercopithecus aethiops kidney fibroblasts (CV-1) were a kind gift from the Helenius laboratory and grown in DMEM without phenol red, supplemented with $10 \%$ fetal bovine serum (FBS) and GlutaMAX (all ThermoFisher Scientific) at $37^{\circ} \mathrm{C}$ in a $\mathrm{CO}_{2}$-controlled humidified incubator. Stably transfected GPI-GFP CV-1 cells were maintained with $50 \mu \mathrm{g} \mathrm{ml}^{-1}$ Geniticin (ThermoFisher Scientific) supplementing the culture medium. For transient transfection, CV-1 cells at $70-100 \%$ confluency were electroporated with the Neon
Transfection System according to the supplier's protocol for COS-7 cells. Cells were plated on glass-bottom dishes and imaged in imaging medium (Fluorobrite DMEM, 10\% FBS, GlutaMAX, 10-30 mM HEPES; ThermoFisher Scientific) 16-48 $\mathrm{h}$ after transfection.

Particle functionalization and live-cell experiments. For live-cell experiments targeting GFP or YFP, LaG-16 anti-GFP nanobodies ${ }^{39}$ were recombinantly produced $^{40}$ and labeled. For the labeling reaction ${ }^{41}$, $a$-Biotin- $\omega$-(succinimidyl propionate)-24(ethylene glycol) (Iris Biotech) was added to the nanobody at twofold molar excess. After purification via $7 \mathrm{kDa}$ MWCO Zeba Spin Desalting Columns (ThermoFisher Scientific), the labeling efficiency was estimated to be 0.06 dye per protein by labeling with Alexa Fluor 647 carboxylic acid, succinimidyl ester in parallel. The FMNPs were mixed with the biotinylated nanobody in a 10:1 to $1: 2$ 

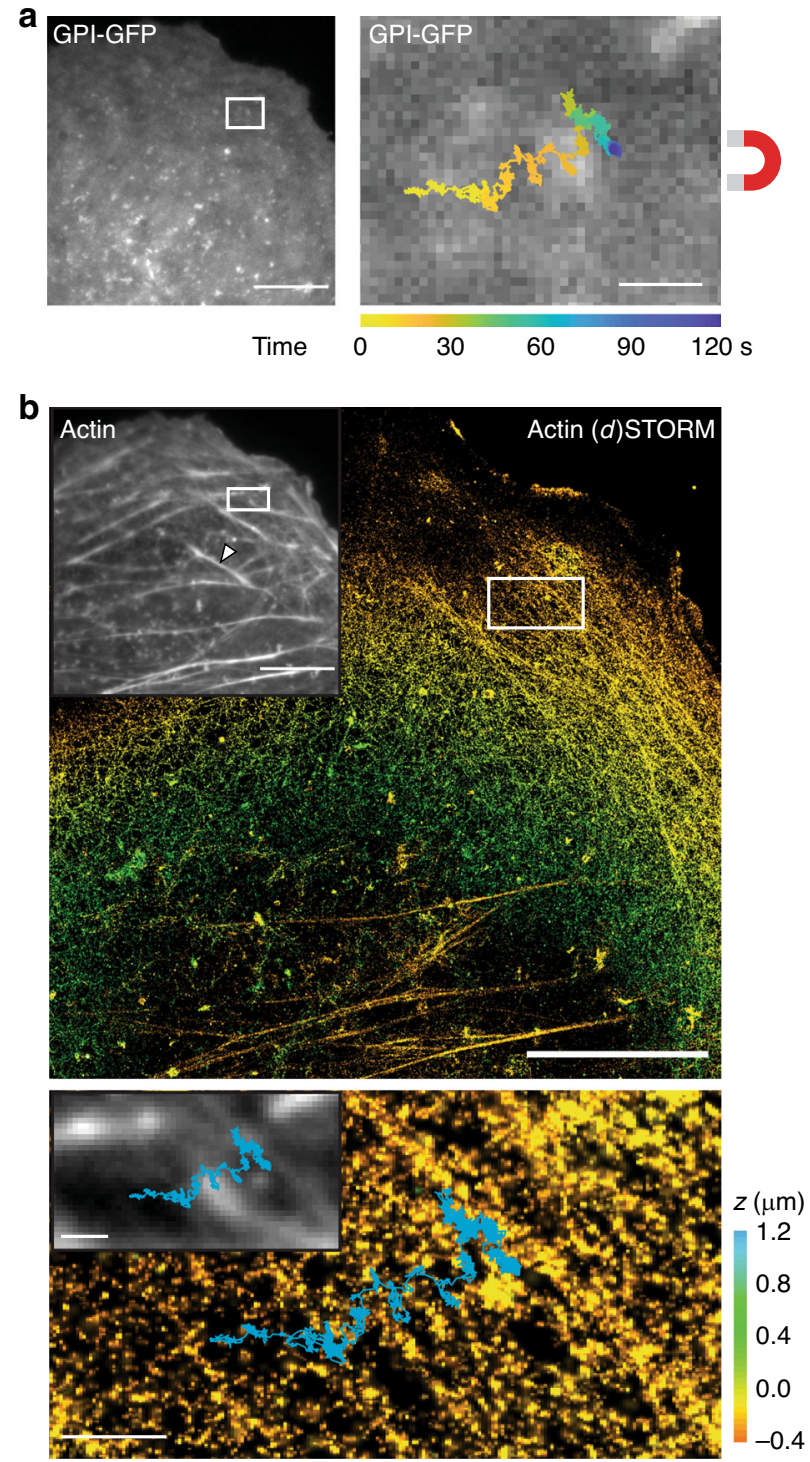

Fig. 4 Correlation of FMNP-steered protein movement and the underlining F-actin cytoskeleton. a Live fluorescence image of the membranous GPI-GFP signal in a CV-1 cell. Scale bar is $10 \mu \mathrm{m}$. Right: Trajectory of a FMNP bound to GPI-GFP and pulled by the magnetic needle overlaid onto the GPI-GFP signal. Scale bar is $1 \mu \mathrm{m}$. b Top: Phalloidin-AF647 stained $\mathrm{F}$-actin in the same cell as in a after fixation, imaged by conventional epifluorescence microscopy (inset) and 3D (d)STORM superresolution microscopy (color scale by depth $z$, bottom $0.6 \mu \mathrm{m} z$-slice not included). Arrowhead in the epifluorescence inset indicates a stress fiber, which is excluded in the super-resolution z-slice. Scale bar is $10 \mu \mathrm{m}$. Bottom: Trajectory of FMNP (cyan) overlaid onto the epifluorescence (inset) and (d)STORM image after image registration via fiducials. Scale bar is $1 \mu \mathrm{m}$.

molar ratio, magnetically separated for $1 \mathrm{~h}$ to remove the majority of unlabeled nanobodies, and then resuspended in imaging medium. Living cells were incubated with this suspension at a final concentration of $10^{10}-10^{11}$ particles per $\mathrm{ml}$ in imaging medium for $15 \mathrm{~min}$, washed three to five times with PBS or medium, and imaged in imaging medium.

Microscopy. SLBs were imaged with TIRF illumination. Cells were imaged in epifluorescence illumination because the FMNPs bind to the dorsal cell membrane. Typically for SPT, a single image series of 1,000-10,000 frames was collected with a $5-50 \mathrm{~ms}$ acquisition time $(20-200 \mathrm{~Hz}$, in total $20 \mathrm{~s}$ to $200 \mathrm{~s})$ at a laser-power density of $8-200 \mathrm{~W} \mathrm{~cm}^{-2}$ using the $561 \mathrm{~nm}$ laser line. All experiments were carried out at room temperature to reduce endocytic activity. For the force calibration, longer frame times were recorded ( $100 \mathrm{~ms}$ exposure time with $1-7 \mathrm{~Hz}$ frame rate). For correlative imaging of the cytoskeleton, $100 \mathrm{~nm}$ red fluorescent (580/605) beads (ThermoFisher Scientific) were added as fiduciary markers and cells were fixed immediately after live-cell imaging and stained with phalloidin-Alexa Fluor $647^{33}$. (d)STORM imaging was performed with blinking buffer $(150 \mathrm{mM}$ Tris-HCl, $1.5 \% \beta$-mercaptoethanol, $0.5 \%(\mathrm{v} / \mathrm{w})$ glucose, $0.25 \mathrm{mg} \mathrm{ml}^{-1}$ glucose oxidase and $20 \mathrm{\mu g} \mathrm{ml}^{-1}$ catalase (Sigma Aldrich), $\mathrm{pH} \mathrm{8.8)} \mathrm{at} \mathrm{a} \mathrm{laser-power} \mathrm{density}$ of $4.4 \mathrm{~kW} \mathrm{~cm}^{-2}$ using the $640 \mathrm{~nm}$ laser, acquiring 20,000-40,000 frames with $20 \mathrm{~ms}$ acquisition time ${ }^{42}$. SPT and (d)STORM imaging were performed on a Vutara 352 super-resolution microscope (Bruker) equipped with a $\times 60 / \mathrm{NA} 1.49$ oil immersion TIRF objective (Olympus) and an ORCA Flash 4.0 V2 CMOS (Hamamatsu) at $99 \mathrm{~nm}$ pixel size. The force calibration experiments were performed on an inverted Ti-E TIRF microscope (Nikon) that was equipped with a high-pressure mercury lamp, an Apo TIRF ×60/NA 1.49 oil objective, and an Andor Zyla 4.2 sCMOS camera (Andor Technology) at $2 \times 2$ binning and $217 \mathrm{~nm}$ pixel size. FRAP was performed on an inverted IX71 microscope (Olympus) equipped with a CSU-X1 spinning disk (Yokogawa) and an iLas2 FRAP system (Gataca Systems). A ×60/NA 1.42 oil objective (Olympus) was used and time-lapse image sequences were taken with an ORCA Flash 4.0LT CMOS camera (Hamamatsu).

Data analysis. Analysis of (d)STORM imaging was done in the SRX software (Bruker). The localizations were rendered with a Gaussian distribution by radial precision (Euclidean norm of the precision of the $x$ and $y$ axes calculated as the Cramér-Rao lower bound from the Fisher information of the point spread function). All other images were analyzed with $\mathrm{Fiji}^{3}$. SPT analysis was performed with the MOSAIC Particle Tracker ${ }^{44}$ or with Trackmate ${ }^{45}$. Immobile particles $(D<$ $0.005 \mu \mathrm{m}^{2} \mathrm{~s}^{-1}$ ) and trajectories shorter than 50 frames were discarded. Image registration via fiducials was performed by nonreflective similarity transformation in Matlab (MathWorks) using the built-in function fitgeotrans. Apparent forces were calculated with a Matlab script adapted from Block et al. ${ }^{24}$. Trajectories slower than $0.01 \mu^{2} \mathrm{~s}^{-1}$, shorter than 200 frames and with a displacement smaller than $10 \mu \mathrm{m}$ were discarded. Trajectories and other data were analyzed and plotted with OriginPro (OriginLab). Statistics of the diffusion coefficient were calculated for log $(D)$ due to the log-normal distribution of $D$ and differences between before and after magnetic dragging were tested using a two-tailed paired Student's $t$-test $(t$-statistic $=0.63$, degrees of freedom $=9, P$-value $=0.54)$.

Reporting summary. Further information on research design is available in the Nature Research Reporting Summary linked to this article.

\section{Data availability}

Data supporting the findings of this manuscript are available from the corresponding author upon reasonable request. A reporting summary for this Article is available as a Supplementary Information file. Source data are provided with this paper. The microscopy data that support the findings of this study will be available from https:// github.com/AG-Ewers and from the corresponding author upon request.

\section{Code availability}

The Matlab code for the force extraction is available from https://github.com/ stephanblock/SPT_force_extraction and the Matlab code for the track registration is available from https://github.com/braedyn-au/TrackRegister.

Received: 14 April 2020; Accepted: 4 August 2020; Published online: 26 August 2020

\section{References}

1. Nicolson, G. L. The Fluid-Mosaic Model of Membrane Structure: Still relevant to understanding the structure, function and dynamics of biological membranes after more than 40 years. Biochim. Biophys. Acta Biomembr. 1838, 1451-1466 (2014)

2. Kusumi, A. et al. Dynamic organizing principles of the plasma membrane that regulate signal transduction: commemorating the fortieth anniversary of Singer and Nicolson's Fluid-Mosaic Model. Annu. Rev. Cell Dev. Biol. 28, 215-250 (2012).

3. Bethani, I., Skånland, S. S., Dikic, I. \& Acker-Palmer, A. Spatial organization of transmembrane receptor signalling. EMBO J. 29, 2677-2688 (2010).

4. Finger, F. P. \& Novick, P. Spatial regulation of exocytosis: Lessons from yeast. J. Cell Biol. 142, 609-612 (1998).

5. Palm, W. \& Thompson, C. B. Nutrient acquisition strategies of mammalian cells. Nature 546, 234-242 (2017).

6. Sourjik, V. \& Armitage, J. P. Spatial organization in bacterial chemotaxis. EMBO J. 29, 2724-2733 (2010).

7. Rozario, T. \& DeSimone, D. W. The extracellular matrix in development and morphogenesis: a dynamic view. Dev. Biol. 341, 126-140 (2010). 
8. Jacobson, K., Liu, P. \& Lagerholm, B. C. The lateral organization and mobility of plasma membrane components. Cell 177, 806-819 (2019).

9. Sako, Y. \& Kusumi, A. Barriers for lateral diffusion of transferrin receptor in the plasma membrane as characterized by receptor dragging by laser tweezers: fence versus tether. J. Cell Biol. 129, 1559-1574 (1995).

10. Sako, Y., Nagafuchi, A., Tsukita, S., Takeichi, M. \& Kusumi, A. Cytoplasmic regulation of the movement of E-cadherin on the free cell surface as studied by optical tweezers and single particle tracking: corralling and tethering by the membrane skeleton. J. Cell Biol. 140, 1227-1240 (1998).

11. Lee, N. \& Hyeon, T. Designed synthesis of uniformly sized iron oxide nanoparticles for efficient magnetic resonance imaging contrast agents. Chem. Soc. Rev. 41, 2575-2589 (2012).

12. Zhang, H. et al. Magnetic nanoparticles based cancer therapy: current status and applications. Sci. China Life Sci. 61, 400-414 (2018).

13. Neuman, K. C. \& Nagy, A. Single-molecule force spectroscopy: Optical tweezers, magnetic tweezers and atomic force microscopy. Nat. Methods $\mathbf{5}$, 491-505 (2008).

14. Collins, C. et al. Localized tensional forces on PECAM-1 elicit a global mechanotransduction response via the integrin-RhoA pathway. Curr. Biol. 22, 2087-2094 (2012).

15. Chronopoulos, A. et al. Syndecan-4 tunes cell mechanics by activating the kindlin-integrin-RhoA pathway. Nat. Mater. 19, 669-678 (2020).

16. Zaner, K. S. \& Valberg, P. A. Viscoelasticity of F-actin measured with magnetic microparticles. J. Cell Biol. 109, 2233-2243 (1989).

17. Monzel, C., Vicario, C., Piehler, J., Coppey, M. \& Dahan, M. Magnetic control of cellular processes using biofunctional nanoparticles. Chem. Sci. 8, 7330-7338 (2017).

18. Sauer, A. M. et al. Dynamics of magnetic lipoplexes studied by single particle tracking in living cells. J. Control. Release 137, 136-145 (2009).

19. Etoc, F. et al. Subcellular control of Rac-GTPase signalling by magnetogenetic manipulation inside living cells. Nat. Nanotechnol. 8, 193-198 (2013).

20. Etoc, F. et al. Magnetogenetic control of protein gradients inside living cells with high spatial and temporal resolution. Nano Lett. 15, 3487-3494 (2015).

21. Seo, D. et al. A mechanogenetic toolkit for interrogating cell signaling in space and time. Cell 165, 1507-1518 (2016).

22. Huang, H., Delikanli, S., Zeng, H., Ferkey, D. M. \& Pralle, A. Remote control of ion channels and neurons through magnetic-field heating of nanoparticles. Nat. Nanotechnol. 5, 602-606 (2010).

23. Mannix, R. J. et al. Nanomagnetic actuation of receptor-mediated signal transduction. Nat. Nanotechnol. 3, 36-40 (2008).

24. Block, S., Fast, B. J., Lundgren, A., Zhdanov, V. P. \& Höök, F. Twodimensional flow nanometry of biological nanoparticles for accurate determination of their size and emission intensity. Nat. Commun. https://doi. org/10.1038/ncomms12956 (2016).

25. Evans, E. Probing the relation between force-lifetime-and chemistry in single molecular bonds. Annu. Rev. Biophys. Biomol. Struct. 30, 105-128 (2001).

26. Goswami, D. et al. Nanoclusters of GPI-anchored proteins are formed by cortical actin-driven activity. Cell 135, 1085-1097 (2008).

27. Weigel, A. V., Simon, B., Tamkun, M. M. \& Krapf, D. Ergodic and nonergodic processes coexist in the plasma membrane as observed by single-molecule tracking. Proc. Natl Acad. Sci. USA 108, 6438-6443 (2011).

28. Mueller, V. et al. STED nanoscopy reveals molecular details of cholesteroland cytoskeleton-modulated lipid interactions in living cells. Biophys. J. 101, 1651-1660 (2011).

29. Fujiwara, T. K. et al. Confined diffusion of transmembrane proteins and lipids induced by the same actin meshwork lining the plasma membrane. Mol. Biol. Cell 27, 1101-1119 (2016).

30. Albrecht, D. et al. Nanoscopic compartmentalization of membrane protein motion at the axon initial segment. J. Cell Biol. 215, 37-46 (2016).

31. Sungkaworn, T. et al. Single-molecule imaging reveals receptor-G protein interactions at cell surface hot spots. Nature 550, 543-547 (2017).

32. Freeman, S. A. et al. Transmembrane pickets connect cyto- and pericellular skeletons forming barriers to receptor engagement. Cell 172, 305-317.e10 (2018).

33. Xu, K., Babcock, H. P. \& Zhuang, X. Dual-objective STORM reveals threedimensional filament organization in the actin cytoskeleton. Nat. Methods $\mathbf{9}$, 185-188 (2012).

34. Bharde, A. A. et al. Magnetic nanoparticles as mediators of ligand-free activation of EGFR signaling. PLOS ONE 8, https://doi.org/10.1371/journal. pone.0068879 (2013).

35. Clausen, M. P. \& Lagerholm, B. C. Visualization of plasma membrane compartmentalization by high-speed quantum dot tracking. Nano Lett. 13, 2332-2337 (2013).

36. Reina, F. et al. Complementary studies of lipid membrane dynamics using iSCAT and super-resolved fluorescence correlation spectroscopy. J. Phys. D Appl. Phys. 51, 235401 (2018).

37. Madariaga-Marcos, J. et al. Force determination in lateral magnetic tweezers combined with TIRF microscopy. Nanoscale 10, 4579-4590 (2018).
38. Kukura, P. et al. High-speed nanoscopic tracking of the position and orientation of a single virus. Nat. Methods 6, 923-927 (2009).

39. Fridy, P. C. et al. A robust pipeline for rapid production of versatile nanobody repertoires. Nat. Methods 11, 1253-1260 (2014).

40. Fabricius, V., Lefèbre, J., Geertsema, H., Marino, S. F. \& Ewers, H. Rapid and efficient C-terminal labeling of nanobodies for DNA-PAINT. J. Phys. D. Appl. Phys. 51, 474005 (2018).

41. Platonova, E., Ewers, H. \& Winterflood, C. M. A simple method for GFP- and RFP-based dual color single-molecule localization microscopy. ACS Chem. Biol. 10, 1411-1416 (2015).

42. Ries, J., Kaplan, C., Platonova, E., Eghlidi, H. \& Ewers, H. A simple, versatile method for GFP-based super-resolution microscopy via nanobodies. Nat. Methods 9, 582-584 (2012).

43. Pietzsch, T. et al. Fiji: an open-source platform for biological-image analysis. Nat. Methods 9, 676-682 (2012)

44. Sbalzarini, I. F. \& Koumoutsakos, P. Feature point tracking and trajectory analysis for video imaging in cell biology. J. Struct. Biol. 151, 182-195 (2005).

45. Tinevez, J. Y. et al. TrackMate: an open and extensible platform for singleparticle tracking. Methods 115, 80-90 (2017).

\section{Acknowledgements}

We thank Prof. Blankenstein and Prof. Willemsky for lending the micromanipulator and the engineering workshop of the Institute of Chemistry of the Freie Universität Berlin for the custom fabrication of adaptors for the micromanipulator. The GPI-GFP and TfRGFP plasmids were a kind gift from the Helenius laboratory, the L-YFP-GT46 plasmid was a kind gift of Patrick Keller. We thank Areeya Chawandee, Raluca Groza, and Dr. Vladimira Petrakova for testing reagents or performing initial experiments. The work was funded by Deutsche Forschungsgemeinschaft (DFG) through SFB 958, SFB 756, and TRR186. We would like to acknowledge the assistance of the Core Facility BioSupraMol supported by the DFG. We acknowledge technical support from Andrea Senge, Claire Schlack, Reinmar Undeutsch, Jonathan Lefèbre, Valentin Fabricius, and Carolin Knappe

\section{Author contributions}

H.E. designed research; J.H.L. and P.S.-O. performed research; B.A., J.R., and S.B. con tributed new reagents or analytic tools; J.H.L. analyzed data; S.B. analyzed force calibration data; and J.H.L and H.E. wrote the manuscript with input from all authors.

\section{Funding}

Open access funding provided by Projekt DEAL.

\section{Competing interests}

The authors declare no competing interests.

\section{Additional information}

Supplementary information is available for this paper at https://doi.org/10.1038/s41467 020-18087-3.

Correspondence and requests for materials should be addressed to H.E.

Peer review information Nature Communications thanks Young-wook Jun and the other, anonymous, reviewers for their contributions to the peer review of this work. Peer review reports are available.

Reprints and permission information is available at http://www.nature.com/reprints

Publisher's note Springer Nature remains neutral with regard to jurisdictional claims in published maps and institutional affiliations.

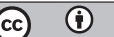

Open Access This article is licensed under a Creative Commons Attribution 4.0 International License, which permits use, sharing, adaptation, distribution and reproduction in any medium or format, as long as you give appropriate credit to the original author(s) and the source, provide a link to the Creative Commons license, and indicate if changes were made. The images or other third party material in this article are included in the article's Creative Commons license, unles indicated otherwise in a credit line to the material. If material is not included in the article's Creative Commons license and your intended use is not permitted by statutory regulation or exceeds the permitted use, you will need to obtain permission directly from the copyright holder. To view a copy of this license, visit http://creativecommons.org/ licenses/by/4.0/

(c) The Author(s) 2020 